\title{
NeuroImage
}

ELSEVIER

\section{Predictability modulates the affective and sensory-discriminative neural processing of pain}

\author{
Katrina Carlsson, ${ }^{\mathrm{a}, *}$ Jesper Andersson, ${ }^{\mathrm{a}}$ Predrag Petrovic, ${ }^{\mathrm{a}}$ Karl Magnus Petersson, ${ }^{\mathrm{a}, \mathrm{b}, \mathrm{c}}$ \\ Arne Öhman, ${ }^{\mathrm{a}}$ and Martin Ingvar ${ }^{\mathrm{a}}$ \\ ${ }^{a}$ Department of Clinical Neuroscience, MRC N8, Karolinska Institute and Hospital, SE-171 76 Stockholm, Sweden \\ ${ }^{\mathrm{b}}$ F.C. Donders Center, Radboud University Nijmegen, The Netherlands \\ ${ }^{\mathrm{c}}$ Centre for Intelligent Systems, Universidade do Algarve, Faro, Portugal
}

Received 4 July 2005; revised 7 April 2006; accepted 14 May 2006

Available online 24 July 2006

\begin{abstract}
Knowing what is going to happen next, that is, the capacity to predict upcoming events, modulates the extent to which aversive stimuli induce stress and anxiety. We explored this issue by manipulating the temporal predictability of aversive events by means of a visual cue, which was either correlated or uncorrelated with pain stimuli (electric shocks). Subjects reported lower levels of anxiety, negative valence and pain intensity when shocks were predictable. In addition to attenuate focus on danger, predictability allows for correct temporal estimation of, and selective attention to, the sensory input. With functional magnetic resonance imaging, we found that predictability was related to enhanced activity in relevant sensory-discriminative processing areas, such as the primary and secondary sensory cortex and posterior insula. In contrast, the unpredictable more aversive context was correlated to brain activity in the anterior insula and the orbitofrontal cortex, areas associated with affective pain processing. This context also prompted increased activity in the posterior parietal cortex and lateral prefrontal cortex that we attribute to enhanced alertness and sustained attention during unpredictability.
\end{abstract}

(c) 2006 Elsevier Inc. All rights reserved.

Keywords: Predictability; Prediction; Anticipation; Pain; Anxiety; Imaging; fMRI; Neuroimaging

\section{Introduction}

A sensation of pain typically causes emotional distress. Pain signals a threat to bodily integrity and can be analyzed in framework of homeostatic mechanisms. It reflects one of several afferent modes (e.g., itch, tickle, sensual touch, vasomotor flush, hunger, thirst) that convey information about the state of the body to the brain, and this information might provide important input to

\footnotetext{
* Corresponding author. Fax: +46851773266.

E-mail address: katrina.carlsson@ens.ki.se (K. Carlsson).

Available online on ScienceDirect (www.sciencedirect.com).
}

the neural systems supporting emotion, feelings, and self-awareness (Craig, 2002). In the present study, we explore the interplay between pain, affect, and attentional processes in the context of pain stimuli that are predictable or unpredictable.

The nature of pain is reflected in both sensory-discriminative and affective components (Melzack and Casey, 1968). The sensory-discriminative system includes thalamic nuclei that mediate afferent information to the primary and secondary sensory cortices (SI and SII, respectively) and the posterior insula, which provides an interoceptive context as well as an interface to the affective system (Craig, 2002). The latter includes the anterior insula as a central structure and in addition the anterior cingulate and the orbitofrontal cortices (ACC and OFC, respectively) (Craig, 2002; Rainville, 2002). Singer et al. (2004) reported an interesting dissociation between these systems. Whereas both the sensory-discriminative and the affective systems were activated in a participant receiving actual pain stimulation, the affective, but not the sensory-discriminative, system was empathically activated in the participant observing a signal indicating that an emotionally close partner was receiving the pain stimulus.

Several lines of evidence suggest that interoceptive systems and the anterior insula play a central role in the affective system. First, the anterior insula activity is more closely correlated with subjective magnitude ratings of temperature than with physical increases in temperature (Craig, 2002). Second, visceral stimulation through inflation of a balloon in the esophagus results in insular activation (Aziz et al., 2000). This insular activity, as well as reported discomfort, is enhanced in a negative emotional context (Phillips et al., 2003b). Third, the accuracy in judgment of heartbeat timing is positively correlated with insular activity as well as with indices of negative emotion, providing support for its role in interoceptive awareness (Critchley et al., 2004). Fourth, perception and mapping of bodily states have since James (1894) and Lange (1922) been regarded as central for emotional experience (Mesulam and Mufson, 1985; Damasio et al., 2000; 
Craig, 2002). Accordingly, the anterior insula has consistently been implicated in studies with emotional manipulations (e.g., Phillips et al., 2003a; Carlsson et al., 2004) and specifically associated with the emotional component of pain (Singer et al., 2004).

The orbitofrontal cortex (OFC) has also been given a central position in affective pain processing. This region has a wide range of functions such as representing the value of reinforcing and punishing stimuli (Rolls, 2004). The OFC also responds to breaches in cue-target associations (Nobre et al., 1999), to the variability in response-reinforcement contingencies (Elliott et al., 2000) as well as to aversion prediction error signaling (Seymour et al., 2005). The ventral striatum, which is anatomically and functionally linked to the OFC (Selemon and Goldman-Rakic, 1985), has been suggested to support prediction-error processing, that is, violations of reward expectations, as well as attention to temporal structure (Schultz, 2002; Coull et al., 2004).

The context in which the pain is experienced has previously been shown to influence pain processing (Rainville et al., 1997; Petrovic et al., 2002). For example, subjects anticipating painful stimuli of high intensity report higher anxiety levels in anticipation of the stimulus, and higher pain intensity ratings at stimulus impact, compared to when they anticipate a stimulus of low intensity, even if the intensity in fact was constant (Ploghaus et al., 2001). Similarly, the threat of an electric stimulus increases the anxiety levels as well as the pain reactivity. In contrast to the threat of an electric shock, the exposure to three consecutive electric stimuli has the opposite effect: a decrease in pain reactivity is observed (Rhudy and Meagher, 2000). This reaction to immediate aversive stimuli can be characterized as a fear response, which mobilizes the organism to prepare and, if possible, take rapid action to remove the pain (Öhman, 2000b). Indeed, fear may be viewed as a homeostatic "error signal" conveying a threat to bodily integrity that motivates restoring the homeostatic balance by escaping from or inhibiting the pain stimulus. Thus, fear mobilizes the organism to take action here and now, and it has been suggested that fear and pain can be regarded as mutually inhibiting states, of which fear has priority when it comes to promoting fight and flight (Bolles and Fanselow, 1980). Anxiety, on the other hand, can be characterized as a future-oriented aversive state in the face of an uncertain and potentially uncontrollable threat (Öhman, 2000a). In terms of the homeostatic model, anxiety will prevail when pain reflects a long-term threat to homeostatic balance, probably reflected in salient interoceptive activation. Therefore, anxiety will be the dominating emotion when pain stimulation is uncontrollable and protracted. In such situations, it may be more appropriate to increase vigilance, environmental scanning and arousal, typical reactions observed in conditions of anxiety, with a consequent increase in pain reactivity (Bolles and Fanselow, 1980).

Behavioral control is a primary modulator of the impact of nociceptive input because it provides an avenue for impacting on the expected consequences of the aversive input (Maier and Watkins, 1998). Accordingly, numerous studies have demonstrated that control reduces the subjective experience of stress as well as the corresponding ratings of pain (Miller, 1980). Whereas control typically refers to the capability to do something in order to change a situation, the ability to predict implies knowledge about the relationship between events in a given environment ${ }^{1}$. Similar to control, prediction can modulate the extent to which aversive stimuli induce stress and anxiety (Miller, 1981). Predictability offers the possibility to develop a model of an aversive situation that specifies what can (and cannot) be expected. As a consequence there is potentially less surprise and focus on danger in an aversive situation, which in turn reduces anxiety as well as anticipatory and impact arousal (Berlyne et al., 1960).

Predictable pain stimuli entail a Pavlovian fear conditioning contingency in which the predictive cue (whatever its nature) serves as a conditioned stimulus and the pain stimulus as an unconditioned stimulus. As a result of this contingency, conditioned fear to the cue should be expected, because it is regularly followed by the painful unconditioned stimulus. In a functional perspective (Domjan, 2005) this conditioned fear response will serve to modulate the impact of the pain stimulus, through active avoidance if possible, or though the inhibitory effect of fear on pain (cf., Bolles and Fanselow, 1980) if the situation precludes active responses. Furthermore, the flip side of providing information about when the pain is due, is that the absence of the predictive cue denotes safety (Seligman, 1968). In an unpredictable situation, on the other hand, there are no designated safe periods, if the temporal distribution of pain stimuli is programmed to be random. With such a procedure, conditioning to the context, making the whole situation aversive, should be expected. In such an aversive situation, which lacks specific fear-inducing cues and means to influence the pain stimulus, the resulting emotional state is better described as anxiety than fear (e.g., Öhman, 2000a). Moreover, the emotional states generated in the predictable and unpredictable situations might have different brain substrates.

Predictable and unpredictable situations engender different psychological processes in other respects than conditioning. For example, the conditions for attention deployment are different with predictable and unpredictable stimuli. In the predictable case, selective attention can be precisely directed to the external stimuli, which is likely to result in increased processing in the brain regions that process the specific sensory input to which attention is directed (Rees et al., 1997; Carlsson et al., 2000). In the unpredictable conditions, on the other hand, stimulation cannot be anticipated, and hence attention cannot be selectively timed to the stimuli to the same degree as when they are predictable. However, as generating anxiety, unpredictable conditions are likely to induce a bias for detecting threat (e.g., Mogg and Bradley, 1998), which might activate brain circuits for sustained attention (Pardo et al., 1991).

Predictability can be differentiated in at least two types. The first involves knowing the conditions under which the event will occur (contingency predictability), and the second knowing what the event will be like (what-kind-of-event-predictability) (Miller, 1981). In the present study, the subjects were well acquainted with the nature and location of the stimuli through a procedure of choosing appropriate intensity levels. We designed experiments in which we varied the intensity and the temporal predictability of brief electric shocks. In a pilot experiment, the electrical shocks

\footnotetext{
${ }^{1}$ Predictability in a precise sense is a property of the phenomenon to be predicted, while predictive capacity refers to a property of the system that attempts to predict. A more complex sequence may not be detected by the cognitive system even though the sequence contains a structure, that is, is predictable. The design of the predictable conditions in this study is simple, and the stimuli may readily be predicted. For simplicity, we therefore refer to predictability as including also the predictive capacity.
} 
were presented with a regular rhythm or randomly. In the main experiment of the present study, shocks were preceded by a visual cue in the predictable conditions, and in the unpredictable conditions the cue and the shocks were randomly related. In other words, we manipulated the degree of contingency predictability of a somatosensory stimulus of high or low intensity.

The subjects received the same amount of nociceptive input in the predictable and unpredictable conditions, with the difference that they either could or could not predict when to expect the shocks. The primary aim of this functional magnetic resonance imaging (fMRI) study was to delineate functional neuroanatomical systems related to sensory-discriminative and affective aspects of pain as modulated by predictability and related contexts of anxiety, fear conditioning and attention. For the majority of the subjects, we expected the unpredictability of aversive events to result in an increase in subjective anxiety experience and negative valence with corresponding activity in affective neural processing areas such as the anterior insula and the OFC. We hypothesized predictability, on the other hand, to allow for direction of selective attention and to correlate with increased activity in areas supporting sensory-discriminatory processes, such as the SI, SII and the posterior insula.

\section{Materials and methods}

\section{Participants}

Ten healthy subjects ( $3 \mathrm{~F} / 7 \mathrm{M}$, right handed, non-smoking, mean age 24 years, range 21-34 years) participated in the pilot study. In the main experiment, thirteen healthy subjects participated. Four of these subjects made ratings that were inconsistent between series and were excluded from further analysis. The remaining nine subjects $(5 \mathrm{~F} / 4 \mathrm{M}$, right handed, non-smoking, mean age 25 years, range 21-32 years) were included in the statistical analysis of the behavioral and functional imaging data. All subjects gave their informed consent, and the procedures were approved by the local ethical committee at the Karolinska Hospital.

\section{Nociceptive stimulus delivery and subjective rating}

The experimental protocol for both the pilot and main experiment was defined as a multi-session blocked fMRI paradigm with four sessions, each consisting of four main conditions. Before entering the scanner, the subjects chose two levels of a 70-ms electrical shock stimulation. The shocks were delivered via surface ECG electrodes placed with approximately 4-cm distance from one another on the palmar aspect of the right wrist. Both levels, high (HI) and low (LO), were set in co-operation with the subject. They were instructed to choose the high level so that it was painful and of maximum intensity of what they accepted to endure but clearly within the limit of what would make the participation in the experiment an acceptable experience. The low level (LO) was set so that they without doubt could detect the stimulus but as low as possible. They were told that they could adjust the levels between any of the four sessions. The power supply for the electric stimulation was located outside the camera room. To minimize interference with the scanner, the electrical current applied to the subject was filtered with a low-pass filter. In both the pilot and main experiment, the subjects performed ratings between each of the four scanning sessions. They rated experienced valence, intensity and anxiety associated with each condition. Zero equaled no unpleasantness/pain intensity/anxiety and 100 equaled the highest imaginable unpleasantness/pain intensity/anxiety. This rating procedure was done orally via an intercom system.

\section{Pilot experiment}

The subjects watched a screen from a supine position in the camera via a binocular. They were asked to pay attention to the visual instructions presented on the screen, to try to fix their gaze in the center of the screen and at no time during the sessions close their eyes more than for normal blinking. Somatosensory stimulations were delivered in 20-s blocks, each containing 10 stimuli. In between each block, there were $10 \mathrm{~s}$ of rest. Information about the upcoming blocks of stimuli and rest was shown for $2 \mathrm{~s}$, which made the actual period between the somatosensory stimulations $14 \mathrm{~s}$. A fixation cross was shown at all times except during the instructions. The instructions contained information whether the somatosensory stimuli in the upcoming block were to be of HI or LO intensity and whether they would be delivered in a rhythmic (RHY) or randomized (RA) way. The rhythmic stimuli were programmed to come every $2 \mathrm{~s}$, and the randomized stimuli came in a pseudorandomized fashion every $2 \mathrm{~s}$ with a $1.8 \mathrm{~s}$ jitter. The subjects were uninformed about the interstimulus interval. The subjects completed four sessions containing three blocks of each of the four conditions RA_HI, RA_LO, RHY_HI, and RHY_LO.

\section{Main experiment}

In the same basic setup as for the pilot experiment, the instructions were shown for $2 \mathrm{~s}$, and there were $4 \mathrm{~s}$ of rest in between each $30 \mathrm{~s}$ train of stimuli, making the actual resting period between the blocks of shocks $8 \mathrm{~s}$ (Fig. 2). Each block of stimuli consisted of 6 shocks and 6 colored squares in a pseudorandomized sequence (every $4 \mathrm{~s} \pm 1.8 \mathrm{~s}$ ). Instructions displayed on the screen contained information of whether an upcoming train of stimuli was to be of HI or LO intensity and whether the somatosensory stimuli would be uncorrelated (U) or preceded by and thus correlated (C) to a 0.5 -s visual cue. In the $\mathrm{C}$ conditions, the shock immediately followed the visual cue. The visual cue consisted of a $20-\mathrm{cm}$ color square, either green for the correlated blocks or blue for the uncorrelated blocks. A fixation cross was displayed at the times between instructions/squares. Subjects completed four sessions, each containing three blocks of the four active conditions C_HI, C_LO, U_HI and U_LO. Specific sequences did recur over sessions so that a sequence structure was repeated four times for each subject, however, all twelve of the sequence structures within a session was unique. Also, the order in which the conditions were presented was balanced between sessions and subjects.

All behavioral data were statistically tested with analyses of variance (ANOVAs), in which predictability (rhythm for pilot, and correlation to visual cue for main experiment) and level of intensity served as factors in $2 \times 2$ factorial designs. Tukey's honestly difference (HSD) tests were used as follow-up test when interac-tions were significant.

\section{fMRI data acquisition}

Imaging was performed on a 1.5-T GE Signa Echospeed MR scanner with a standard circular head coil. Foam pads was used to help subject to prevent head movements. Functional images were obtained with a gradient echo-EPI sequence (repetition time, $4.2 \mathrm{~s}$; echo time, $40 \mathrm{~ms}$; flip angle, $90^{\circ}$; voxel size $3.5 \times 3.5 \times 3.0 \mathrm{~mm}$; 
slice gap, $0.5 \mathrm{~mm}, 42$ axial sections). In the pilot experiment, each session was $7 \mathrm{~min}$ long and contained 100 volumes. In the main experiment, the sessions were $7 \mathrm{~min} 36 \mathrm{sec}$ long containing 108 volumes. Stimulus presentation was synchronized by means of using a trigger pulse for each image acquisition from the MR scanner. There were technical problems with this synchronization during two of the sessions for one subject and one session for another subject, and these sessions were hence excluded from the analysis of the data. To reduce magnetic saturation effects, each session began with three dummy scans that were discarded in the statistical analysis.

\section{Image preprocessing and statistical analysis}

All image processing and statistical analysis was performed using SPM2 (www.fil.ion.ucl.ac.uk/spm/spm2.html). First all images for each subject were realigned to the first scan of the first session, corrected for movement-by-susceptibility distortion interactions (Andersson et al., 2001) and re-sampled using a spline-interpolation kernel. A slice timing correction was performed to the model slice to correct for the sequential sampling of the brain in the slice direction. Spatial normalization was based on finding the warping parameters between the mean EPI volume of a given subject and the SPM2 EPI template, an approximate Talairach space (Talairach and Tournous, 1988; Ashburner and Friston, 1999), and applying these parameters to all the individual EPI images. Finally, the images were spatially smoothed using a 12-mm FWHM Gaussian kernel.

Statistical modeling was done using the general linear model (Friston et al., 1995). Each trial-type was modeled as a box-car stimulus function convolved with a canonical hemodynamic response function (HRF) and its temporal derivative. There were four trial-types of interest (correlated high intensity (C_HI), correlated low intensity (C_LO), uncorrelated high intensity (U_HI) and uncorrelated low intensity (U_LO)) and six trial types of no interest (rest periods and instruction periods preceding the different trial types of interest). High-pass filtering was performed with a cut-off frequency of $252 \mathrm{~s}$. Serial autocorrelations were assumed to conform to an AR(1) model, estimated from the residuals using a restricted maximum likelihood algorithm (Harville, 1977; Friston et al., 2002) and corrected for using prewhitening based on the estimated parameters.

Linear contrasts were used to generate one image per contrast and subject and subsequently subjected to a second-level random effects analysis through a one sample $t$ test summary statistic (Holmes and Friston, 1998). A parametric empirical Bayes approach was used to generate conditional (on the data) parameter estimate maps where each value represented the mode of the posterior distribution of the population effect for that specific contrast (Friston et al., 2002). A shrinkage prior with a zero mean and a standard deviation pooled across all voxels was estimated from the same data. These maps were thresholded so that $p$ (beta $>$ $0 \mid y)>0.975$, meaning that only voxels where the $95 \%$ confidence interval did not include zero were considered.

\section{Results}

\section{Behavioral results}

In the pilot experiment, where predictability was based on rhythmicity, the ten subjects rated anxiety, $F(1,9)=24.84, P<$
0.001, negative valence, $F(1,9)=22.19, P<0.01$ and pain intensity, $F(1,9)=9.88, P<0.05$ higher for the unpredictable, randomly presented shocks. The interactions between predictability (RHY/RA) and level (HI/LO) in anxiety $F(1,9)=49.43, P<$ 0.0001 , negative valence $F(1,9)=28.79, P<0.001$ and intensity $F(1,9)=5.70 P<0.05$ indicated significantly higher effects of rhythmicity in the HI compared to the LO level of stimulation. Tukey's HSD test showed an effect for rhythmicity in the HI level of stimulation, $P<0.001$ for anxiety, $P<0.001$ for negative valence and $P<0.01$ for intensity (see Fig. 1).

In the main experiment, four subjects made ratings that were inconsistent between series and were excluded from further analysis. The pattern of variation was different between subjects, and no trend over time was detectable that suggested that, i.e., learning was part of this variability. The remaining nine subjects rated anxiety $F(1,8)=23.83, P<0.01$ and negative valence $F(1,8)=53.08, P<0.0001$ significantly higher for the $\mathrm{U}$ condition compared to the $\mathrm{C}$ condition. No difference in ratings between the two conditions regarding intensity was found. There was also an interaction in reported anxiety $F(1,8)=20.76, P<0.01$ and negative valence $F(1,8)=177.73, P<0.00001$ between temporal predictability $(\mathrm{U} / \mathrm{C})$ and stimulus level (HI/LO) with a larger increase during HI than LO stimulus intensity. Tukey's HSD test showed an effect for predictability in the HI level of stimulation, $P<0.001$, for anxiety and negative valence (see Fig. 1).

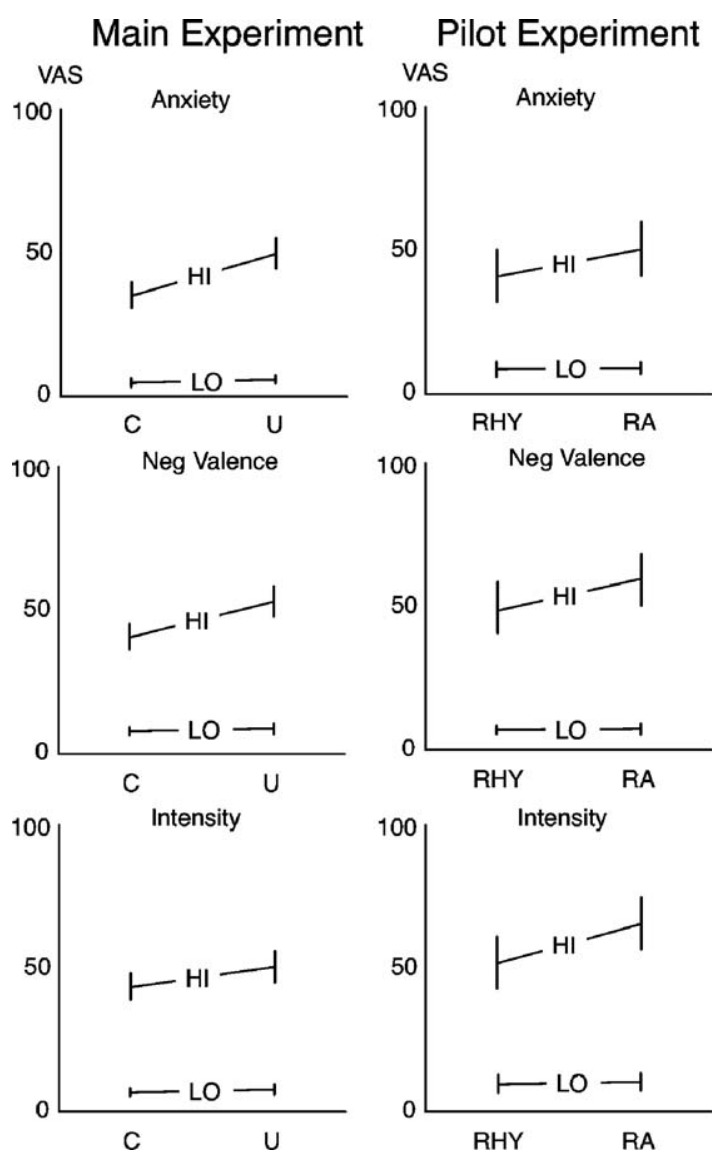

Fig. 1. Graphs depicting the mean \pm standard error of the mean of the ratings of anxiety, negative valence, and pain intensity in the main and the pilot experiment. 


\section{Functional neuroimaging results}

The pilot experiment did not yield any differences between the studied conditions. Hence, only data from the main experiment are reported (see Table 1 and Fig. 2).

$H I$ vs. $L O$

The main effect comparing the high vs. low intensities of the somatosensory stimulation revealed in essence the classical pain matrix (Ingvar, 1999). Activations included the left SI and the bilateral SII, almost all of the ACC from the rostral to the caudal part, the anterior and posterior insula, the periaqueductal gray (PAG), the cerebellum and the visual cortex. The medial OFC was the only active area in the reversed contrast, LO vs. HI (see Table $1 \mathrm{G}$ and $\mathrm{H}$ ).

\section{Uvs. $C$}

Unpredictability (main effect) leads to activations in the right dorsolateral prefrontal cortex (DLPFC) and premotor areas, the right and left posterior parietal cortex (PPC) and the right inferior parietal cortex (IPC) together with the bilateral anterior insula and the right lateral OFC (see Fig. 2, Table 1A).

\section{U_HI vs. C_HI}

The pattern of activation was with some variations, similar in the simple main effects of U_HI vs. C_HI and U_LO vs. C_LO. In U_HI vs. C_HI, the anterior insula activation was confined to the left side and extended into the ventral striatum. The right lateral OFC was activated, but the right fronto-parietal network did not show significant activation in this contrast. Bilateral premotor areas, a left PPC/visual cortex area (BA 19/40) and a caudal ACC area were active (see Fig. 2, Table 1C).

\section{U_LO vs. C_LO}

In the non-painful conditions unpredictability activated the anterior insula, limited to the right side, the right DLPFC and PPC (see Table 1E).

\section{C vs. U}

Allowing for prediction lead to activations the left SI, the SII and posterior insula, both regions bilaterally, the right hippocampus region and the left amygdala region, and the visual cortex (see Fig. 2, Table 1B).

\section{C_HI vs. U_HI}

In the correlated and painful condition, there were activations bilaterally in the posterior insula, and unilaterally in the left visual cortex, the left OFC and in the left hippocampus region (see Table 1D).

\section{C_LO vs. U_LO}

In the corresponding contrast for the low intensity stimulations, the left SI, and bilaterally in the SII, the hippocampus region and the visual cortex were activated (see Table 1F).

Probing for interactions between factors stimulus intensity $x$ predictability revealed no significant differences.

\section{Discussion}

This study was designed to examine cerebral mechanisms underlying the experience of aversive stimuli as altered by predictability. Compared to a condition in which pain stimuli were predictable by means of a visual cue, unpredictable pain stimuli resulted in increased ratings of anxiety and negative valence. The corresponding comparison of the fMRI data showed activations of structures belonging to the affective pain network, including the anterior insula and the lateral OFC. The unpredictable condition required sustained attention, and activity increases were thus noted in the fronto-parietal attentional network. The ventral striatum was also activated in response to the unpredictable aversive events. Predictability of pain stimuli, on the other hand, allows for a more detailed sensory discriminatory processing. Accordingly, activity in the SI and SII and posterior insula was increased. In addition, the anterior medial temporal lobe (amygdala/hippocampal complex) and the visual cortex were more active in the predictable compared to the unpredictable condition.

The visual signal in the $\mathrm{C}$ conditions provided a cue that allowed for correct direction of attentional focus in time and space. It is known that attentional direction towards a stimulus generates faster reaction times (Posner et al., 1980) and an increased neural response in relevant processing areas (Rees et al., 1997; Shulman et al., 1997; Rees and Frith, 1998; Carlsson et al., 2000). The prepared state offered in the $\mathrm{C}$ conditions and the resulting selective attention lead to focused processing of the sensorydiscriminative aspect of the stimuli as reflected in increased activity in the posterior insula, the SI and the SII. While the SI and SII has been described as to provide discriminative aspects of exteroceptive information, the posterior insula has been hypothesized to provide that of interoceptive information, all three regions being arranged topographically (Craig, 2002; Bingel et al., 2003). Small-diameter afferent fibers code for physiological status of various tissues of the body. These fibers terminate on neurons in lamina $I$ in the spinal cord, which in turn project to homeostatic sites in the brainstem and the PAG. The neurons also follow a pathway to the posterior insular cortex and area $3 \mathrm{a}$. In the posterior insula, a topographic modality-selective (pain, temperature, itch, hunger, cardiovascular activity etc.) representation of the interoceptive afferent activity of lamina I is provided. It is this cortical image of interoceptive information in the posterior insula that has been hypothesized to provide discriminative aspects of the pain stimulus (Craig, 2002; Bingel et al., 2003). This conception of pain afference also goes beyond a secluded somatosensory system representation to a specific sensation conveyed by multiple distinct sensory channels.

The electrical stimulus used in this study activates a multitude of sensory afferents (Devor and Seltzer, 1999), which may mean that the detected SI and SII activations should be attributed to general somatosensory rather than to specific pain processing. As the afferent projections to the SI and SII also are arranged topographically, these areas, like the posterior insula, process the discriminative aspects of somatosensory information, but as mentioned, mainly related to exteroceptive information (Craig, 2002).

In addition to directing attention towards a specific external event at a specific time, predictability allows for the structuring a time period into episodes when there is actual danger and other episodes when aversive events are likely not to occur, that is, when one is safe (Seligman, 1968). The response to an external immediate aversive event can be conceptualized as related to a state of fear that mobilizes the organism to take action here and now. The priority of fear over pain to promote fight and flight involves the inhibition of pain (Bolles and Fanselow, 1980). 
Table 1

Local activation maxima

\begin{tabular}{|c|c|c|c|c|}
\hline Region & BA & $\mathrm{CI}$ & Beta & $x, y, z$ \\
\hline \multicolumn{5}{|c|}{ A. Uncorrelated versus correlated ( $U$ vs. $C$ ) } \\
\hline OFC & BA 10 & $0.0361-0.2657$ & 0.1509 & $26,48,-4$ \\
\hline Anterior insula & & $0.0263-0.2568$ & 0.1415 & $36,24,2$ \\
\hline Anterior insula & & $0.0515-0.2800$ & 0.1658 & $-30,18,6$ \\
\hline \multirow[t]{2}{*}{ Premotor cortex } & BA $6 / 8$ & $0.0664-0.2881$ & 0.1773 & $28,20,64$ \\
\hline & BA $6 / 8$ & $0.0443-0.3164$ & 0.1804 & $-34,8,58$ \\
\hline \multirow[t]{3}{*}{ DLPFC } & BA $9 / 45$ & $0.0377-0.2965$ & 0.1671 & $52,18,48$ \\
\hline & BA 45 & $0.0177-0.2628$ & 0.1403 & $58,18,16$ \\
\hline & BA 9 & $0.0319-0.2868$ & 0.1593 & $42,12,22$ \\
\hline \multirow[t]{2}{*}{ PPC } & BA 40 & $0.0418-0.2594$ & 0.1506 & $-30,-54,40$ \\
\hline & BA 40 & $0.0393-0.2945$ & 0.1669 & $46,-48,50$ \\
\hline IPC & BA 42 & $0.0248-0.3204$ & 0.1726 & $58,-54,18$ \\
\hline Cerebellum & & $0.0668-0.2902$ & 0.1785 & $42,-52,-44$ \\
\hline \multicolumn{5}{|c|}{ B. Correlated versus uncorrelated (C vs. U) } \\
\hline Medial frontal lobe & BA 10 & $0.0714-0.3349$ & 0.2031 & $6,52,-2$ \\
\hline Posterior insula & & $0.0475-0.2976$ & 0.1726 & $38,-22,-4$ \\
\hline Posterior insula & & $0.0128-0.2648$ & 0.1388 & $-40,-16,6$ \\
\hline SII & BA $40 / 43$ & $0.0220-0.2869$ & 0.1544 & $54,-14,14$ \\
\hline SII & BA $40 / 43$ & $0.0155-0.2451$ & 0.1303 & $-50,-20,18$ \\
\hline SI & BA $1 / 3$ & $0.0347-0.2697$ & 0.1522 & $-48,-24,62$ \\
\hline SI & BA $1 / 3$ & $0.0003-0.2560$ & 0.1282 & $-54,-36,56$ \\
\hline Hippocampus region & & $0.0500-0.2791$ & 0.1648 & $36,-18,-20$ \\
\hline Amygdala region & & $0.0255-0.2589$ & 0.1402 & $-22,-10,-18$ \\
\hline Visual cortex & BA 18 & $0.1350-0.3906$ & 0.2628 & $6,-86,20$ \\
\hline Cerebellum & & $0.0963-0.4023$ & 0.2493 & $6,-48,-8$ \\
\hline \multicolumn{5}{|c|}{ C. Uncorrelated high versus correlated high (U_HI vs. C_HI) } \\
\hline OFC & BA $10 / 47$ & $0.0244-0.1799$ & 0.1021 & $26,46,-4$ \\
\hline Anterior insula & & $0.0108-0.1697$ & 0.0903 & $-30,26,10$ \\
\hline Putamen/insula & & $0.0406-0.1976$ & 0.1191 & $-30,10,0$ \\
\hline Premotor area & BA $6 / 8$ & $0.0662-0.2302$ & 0.1482 & $-36,10,60$ \\
\hline Premotor area & BA 6 & $0.0542-0.2111$ & 0.1326 & $24,-16,76$ \\
\hline Caudal ACC & BA 31 & $0.0058-0.1675$ & 0.0866 & $12,-12,54$ \\
\hline PCC & BA $23 / 31$ & $0.0108-0.1693$ & 0.0900 & $-4,-26,60$ \\
\hline PPC/visual cortex & BA $19 / 40$ & $0.0578-0.2150$ & 0.1364 & $-40,-80,40$ \\
\hline Pons & & $0.0064-0.1749$ & 0.0907 & $-8,-18,-28$ \\
\hline \multicolumn{5}{|c|}{ D. Correlated high versus uncorrelated high (C_HI vs. U_HI) } \\
\hline $\mathrm{OFC}$ & BA $10 / 11$ & $0.0158-0.1985$ & 0.1072 & $-36,56,-8$ \\
\hline $\mathrm{OFC}$ & BA 47 & $0.0121-0.1818$ & 0.0970 & $-50,46,-10$ \\
\hline Posterior insula & & $0.0544-0.2136$ & 0.1340 & $42,-2,-14$ \\
\hline Posterior insula & & $0.0357-0.1981$ & 0.1169 & $-44,-6,-12$ \\
\hline Posterior insula & & $0.0090-0.1744$ & 0.0917 & $44,-14,6$ \\
\hline Posterior insula & & $0.0129-0.1821$ & 0.0975 & $-44,-22,10$ \\
\hline Hippocampus region & & $0.0164-0.1744$ & 0.0954 & $-16,-14,-20$ \\
\hline Visual cortex & BA $17 / 18$ & $0.0050-0.1783$ & 0.0917 & $-12,-80,14$ \\
\hline Visual cortex & BA 19 & $0.0090-0.1690$ & 0.0849 & $-28,-64,-8$ \\
\hline Cerebellum & & $0.0566-0.2280$ & 0.1423 & $-10,-48,-12$ \\
\hline \multicolumn{5}{|c|}{ E. Uncorrelated low versus correlated low (U_LO vs. C_LO) } \\
\hline DLPFC & BA $8-9$ & $0.0188-0.1631$ & 0.0910 & $50,14,52$ \\
\hline DLPFC & BA44/45 & $0.0155-0.1655$ & 0.0905 & $42,10,24$ \\
\hline DLPFC & BA $6 / 8$ & $0.0153-0.1640$ & 0.0897 & $22,20,60$ \\
\hline DLPFC & BA $6 / 8$ & $0.0067-0.1626$ & 0.0847 & $44,6,40$ \\
\hline Anterior insula & & $0.0247-0.1628$ & 0.0937 & $32,22,-2$ \\
\hline PPC & BA 7 & $0.0284-0.1722$ & 0.1003 & $48,-54,58$ \\
\hline PPC & BA 7 & $0.0238-0.1686$ & 0.0962 & $38,-52,50$ \\
\hline Cerebellum & & $0.0013-0.1416$ & 0.0714 & $40,-52,-46$ \\
\hline \multicolumn{5}{|c|}{ F. Correlated low versus uncorrelated low (C_LO vs. $\left.U_{L} L O\right)$} \\
\hline Medial frontal lobe & BA $9 / 10$ & $0.0105-0.1555$ & 0.0830 & $10,48,0$ \\
\hline Premotor area & BA 6 & $0.0529-0.1885$ & 0.1207 & $-60,0,26$ \\
\hline
\end{tabular}


Table 1 (continued)

\begin{tabular}{|c|c|c|c|c|}
\hline Region & $\mathrm{BA}$ & $\mathrm{CI}$ & Beta & $x, y, z$ \\
\hline Premotor area & BA 6 & $0.0529-0.1916$ & 0.1233 & $-60,-6,24$ \\
\hline SII & BA 43 & $0.0144-0.1621$ & 0.0868 & $52,-10,16$ \\
\hline SII & & $0.0212-0.1696$ & 0.0954 & $-64,-20,30$ \\
\hline $\mathrm{SI} / \mathrm{MI}$ & BA $1 / 4$ & $0.0359-0.1742$ & 0.1050 & $-52,-22,56$ \\
\hline $\mathrm{SI} / \mathrm{MI}$ & BA $1 / 4$ & $0.0215-0.1630$ & 0.0922 & $-34,-24,34$ \\
\hline $\mathrm{SI} / \mathrm{MI}$ & BA $1 / 4$ & $0.0115-0.1553$ & 0.0834 & $-38,-26,60$ \\
\hline $\mathrm{SI} / \mathrm{MI}$ & BA $1 / 4$ & $0.0039-0.1511$ & 0.0774 & $-40,-16,52$ \\
\hline Visual cortex & BA 18 & $0.0277-0.1727$ & 0.1002 & $10,-100,22$ \\
\hline Visual cortex & BA 18 & $0.0008-0.1649$ & 0.0829 & $10,-82,22$ \\
\hline Hippocampus region & & $0.0046-0.1480$ & 0.0763 & $-28,-14,-20$ \\
\hline Hippocampus region & & $0.0143-0.1610$ & 0.0876 & $38,-16,-18$ \\
\hline Cerebellum & & $0.0062-0.1578$ & 0.0820 & $-10,-52,-20$ \\
\hline \multicolumn{5}{|c|}{ G. High versus low (HI vs. LO) } \\
\hline $\mathrm{OFC}$ & BA $47 / 11$ & $0.1217-0.4796$ & 0.3006 & $-34,24,-12$ \\
\hline $\mathrm{OFC}$ & BA 47 & $0.1539-0.4973$ & 0.3256 & $34,18,-16$ \\
\hline Rostral ACC & BA 32 & $0.2288-0.5971$ & 0.4130 & $-2,52,12$ \\
\hline Rostral ACC & BA 32 & $0.2152-0.6203$ & 0.4178 & $2,38,20$ \\
\hline Dorsal ACC & BA 32 & $0.0295-0.4638$ & 0.2466 & $8,16,40$ \\
\hline Dorsal ACC & BA 32 & $0.0590-0.4413$ & 0.2501 & $8,6,40$ \\
\hline Caudal ACC & BA 31 & $0.1887-0.5358$ & 0.3623 & $-10,0,42$ \\
\hline Caudal ACC & BA 31 & $0.2156-0.5373$ & 0.3764 & $-10,-16,44$ \\
\hline Insula/frontal operculum & & $0.2613-0.6548$ & 0.4581 & $50,26,-6$ \\
\hline Insula/frontal operculum & & $0.1777-0.4844$ & 0.3310 & $-50,30,0$ \\
\hline Anterior insula & & $0.1021-0.4496$ & 0.2758 & $36,12,6$ \\
\hline Anterior insula & & $0.1150-0.4639$ & 0.2895 & $-34,10,-4$ \\
\hline Posterior insula & & $0.0448-0.3829$ & 0.2139 & $46,-16,0$ \\
\hline Posterior insula & & $0.1707-0.4934$ & 0.3321 & $-40,-18,-6$ \\
\hline SII & BA 40 & $0.0747-0.4186$ & 0.2466 & $42,-22,20$ \\
\hline SII & BA 40 & $0.1927-0.5309$ & 0.3618 & $-42,-26,24$ \\
\hline SI & BA $1 / 3$ & $0.1250-0.5530$ & 0.3390 & $-28,-30,64$ \\
\hline SI & BA $1 / 3$ & $0.0405-0.5078$ & 0.2742 & $-42,-26,66$ \\
\hline PAG & & $0.0639-0.4149$ & 0.2389 & $6,-32,-28$ \\
\hline PAG & & $0.0868-0.4878$ & 0.2873 & $10,-24,-10$ \\
\hline Visual cortex & & $0.1283-0.5263$ & 0.3273 & $2,-86,10$ \\
\hline Cerebellum & & $0.1092-0.5046$ & 0.3069 & $-14,-58,-20$ \\
\hline \multirow[t]{2}{*}{ Cerebellum } & & $0.1239-0.5559$ & 0.3399 & $16,-46,-18$ \\
\hline & & $0.1656-0.5222$ & 0.3439 & $10,-64,-44$ \\
\hline \multicolumn{5}{|c|}{ H. Low versus High (LO vs. HI) } \\
\hline $\mathrm{OFC}$ & BA 11 & $0.1615-0.4743$ & 0.3179 & $16,42,-20$ \\
\hline $\mathrm{OFC}$ & BA 11 & $0.1727-0.5409$ & 0.3568 & $-18,48,-18$ \\
\hline
\end{tabular}

Note. The $x, y, z$ coordinates refer to the standard space of the EPI template as implemented in SPM2 (an approximate Talairach space (Talairach and Tournoux, 1988)). BA = Brodmann area; $\mathrm{SII}=$ secondary somatosensory cortex; $\mathrm{SI}=$ primary somatosensory cortex; OFC $=$ orbitofrontal cortex; DLPFC $=$ dorsolateral prefrontal cortex; $\mathrm{PPC}=$ posterior parietal cortex; $\mathrm{IPC}=$ inferior parietal cortex; $\mathrm{ACC}=$ anterior cingulate cortex, $\mathrm{PCC}=$ posterior cingulate cortex; $\mathrm{MI}=$ primary motor area; $\mathrm{MTL}=$ medial temporal lobe; $\mathrm{PAG}=$ periaqueductal gray.

Unpredictability, on the other hand, leads to anxiety and increased environmental and somatic scanning that facilitates sensory receptivity (cf. Rhudy and Meagher, 2000) and increases the painful experience. Our experimental paradigm was not primarily designed to delineate the differences between fear and anxiety. However, we speculate that the electric shock following the visual cue in the $\mathrm{C}$ conditions with certainty leads to relatively more specifically timed fear compared to the $U$ conditions, whose unpredictable shocks generated more anxiety (Walker et al., 2003).

Indeed, the correlated conditions not only resulted in lower anxiety ratings, but they also fulfilled requirements for a fear conditioning paradigm in which the visual cue (the green square) reliably predicted a potentially painful shock in the high stimulus intensity condition. Furthermore, the unpredictable $U$ condition can be viewed as a stringent control condition for assessing associative effects of conditioning, because it presented visual (the blue square) and somatosensory stimuli randomly (Rescorla, 1967). Thus, areas that were more active during the $\mathrm{C}$ than the $\mathrm{U}$ condition may reflect associative changes as a result of Pavlovian conditioning. The role of amygdala in fear conditioning has been well documented in lesion studies of both animals (Davis, 1994; LeDoux, 2000) and humans (LaBar et al., 1995), as well as in human functional imaging experiments (e.g., LaBar et al., 1998; Morris et al., 2001; Buchel and Dolan, 2000). As expected from this perspective, in our study, the amygdala was more active in the correlated as opposed to the uncorrelated conditions. Fear conditioning work with rats shows that the conditioned defense response (e.g., freezing) may include analgesia as preparation for a painful unconditioned stimulus (Bolles and Fanselow, 1980; Fanselow and Helmstetter, 1988). That is, the increased activity 

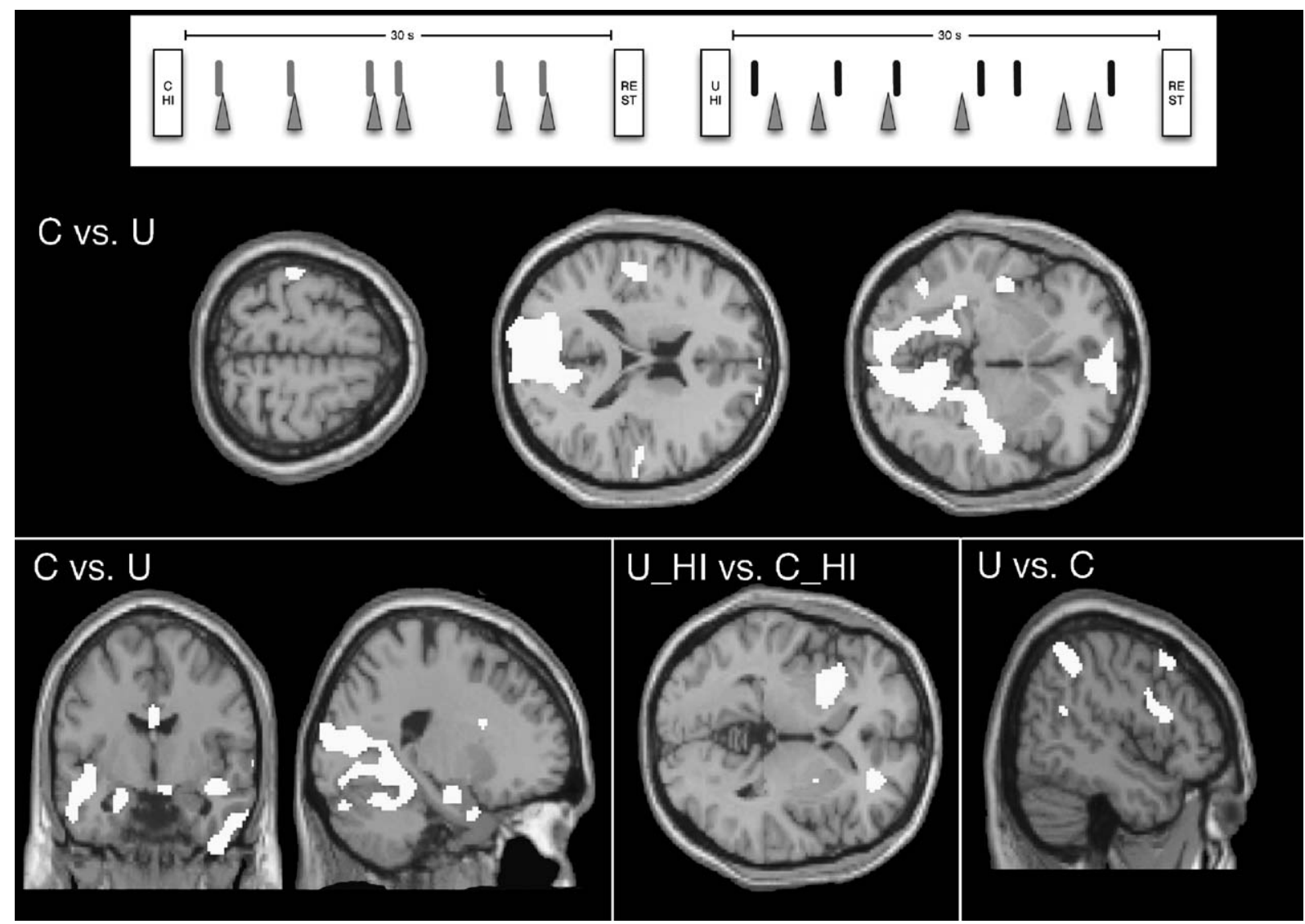

Fig. 2. Top: Experimental design for the main experiment showing the random presentation of visual cues (green (grey bars) and blue (black bars) squares, duration $0.5 \mathrm{~s}$ ) and somatosensory stimuli $(70 \mathrm{~ms})$. In the correlated $(\mathrm{C})$ conditions, the green visual cues preceded the somatosensory stimuli, while the visual and somatosensory stimuli were uncorrelated in the $\mathrm{U}$ condition. The instructions were shown for $2 \mathrm{~s}$, and there were $4 \mathrm{~s}$ of rest in between each 30 -s train of stimuli. Four sessions of $8 \mathrm{~min}$ each contained three blocks of the four active conditions Correlated High (C_HI), Correlated Low (C_LO), Uncorrelated High (U_HI) and Uncorrelated Low (U_LO). (Upper row) Primary sensory cortex $(z=62)$, secondary sensory cortex $(z=14)$ and posterior insula $(z=-4)$ in C vs. U. (Lower left) Left amygdala ( $y=-6, x=-20)$ in C vs. U. (Lower middle) Left anterior insula and putamen and right orbitofrontal cortex ( $z=0)$ in U_HI vs. C_HI. (Lower right) Posterior parietal cortex and dorsolateral prefrontal cortex $(x=48)$ in U vs. C. CI $=90 \%$ for illustrative purposes.

of the amygdala during the correlated conditions in our experiment may be part of a process that dampens the impact of the somatosensory stimuli, thus resulting in decreased ratings of pain and anxiety. The projections of the central nucleus of amygdala to the lateral hypothalamus and the PAG give the amygdala a possibility to modulate autonomic changes, behavioral fear responses as well as analgesia. It is therefore possible that the involvement of amygdala in this type of conditioning may, in addition to play a key role in the associative learning about emotionally salient information, also be part of the downregulating of physiological reactions intrinsic to the improved coping that was possible in the predictable conditions.

The higher anxiety and negative valence ratings during the $U$ conditions activated areas related to affective processing; the anterior insula, the OFC and the ACC (U_HI vs. C_HI). In addition to the sensory-discriminative component, pain processing also contains an affective, motivational aspect (Craig, 2002). The cortical image based on interoceptive information represented in the posterior insula is re-represented in the middle and anterior insula (Craig, 2003). Whereas the posterior insula has been hypothesized to provide the sensory-discriminative aspects of the pain stimulus, the anterior insula with its strong anatomical connections to, for example, the amygdala and the adjacent lateral
OFC, is more important in the affective and autonomic components of pain (Craig, 2002; Bingel et al., 2003). The anterior insula has consistently been activated in studies with emotional manipulations (Phillips et al., 2003a; Carlsson et al., 2004) and specifically associated with the emotional component of pain (Singer et al., 2004). For example, knowing that a loved one experienced pain was sufficient to activate the anterior insula, while activity in the posterior insula was specific to receiving pain (Singer et al., 2004). Functional imaging studies have also provided evidence for the role of the anterior insula in creating a map of the bodily autonomic state (Aziz et al., 2000; Critchley et al., 2004). Thus, the anterior insula is involved in both the mapping of bodily and affective states, two phenomena hypothesized already by James (1894) and Lange (1922) to be closely interlinked. This is consistent with the co-occurrence of the reported higher level of anxiety/negative valence and the anterior insula activity during the $U$ conditions as well as with the conceptualization of pain as a bodily feeling with an inherent association with emotion.

The caudal ACC coordinate of the activation maximum in the unpredictable painful condition (U_HI vs. C_HI) was in a dorsal location $(z=54)$. Some atlases claim that the caudal ACC reaches upwards and includes also this region (Greitz et al., 1991) while in other atlases this location would fall into the supplementary motor 
area. Thus, the denotation of this activation as located within the caudal ACC, may be regarded as somewhat speculative, although it is consistent with the association of this region's with the affective tone of pain processing (Vogt and Pandya, 1987; Buchel et al., 2002).

As the OFC is a key structure in processing reward and aversive information (Schultz et al., 2000; Rolls, 2004), the activation we observed in the $U$ conditions in the lateral OFC can be conceptualized as associated to the more aversive and disturbing unpredictable pain. Another possible interpretation of the role of the OFC in this condition is related to this region's implication in behavioral flexibility and withholding prepared responses (Elliott et al., 2000). For instance, Nobre et al. (1999) reported a corresponding OFC activation in conditions when cues carried invalid predictive information, that is, when expectancies failed, and Seymour et al. (2005) recently reported an activation in the lateral $\mathrm{OFC}$ in response to an aversive prediction error signal.

Identifying patterns and making temporal estimation of stimuli arrival are important components in the anticipation of upcoming events; a key signal being the prediction error in the estimate. A change in sequencing, that is, a violation of expectation, has previously been related to activity in the ventral striatum (Berns et al., 1997; Pagnoni et al., 2002; Schultz, 2002; Seymour et al., 2004; Forkstam and Petersson, 2005). In line with this, we attribute the activation in the ventral striatum during the $\mathrm{U}$ conditions, to attempt to develop a model that would make the pain stimuli more predictable by recruiting areas responsible for learning about temporal cues.

It has been argued that attentional focus and anxiety usually are confounded in studies of effect of anxiety on pain and that it is the attentional focus and not the anxiety that actually lead to increased pain experience (Arntz et al., 1994). We suggest that our study design provided a distinction between anxiety and sustained attention on the one hand and selective attention on the other. Unpredictable aversiveness in the $U$ conditions prompted sustained attention and anxiety, whereas predictability in the $\mathrm{C}$ conditions allowed for less focusing on danger and more selective attention towards somatosensory-discriminative processing as reflected in the increased activity in the posterior insula, the SI and the SII. Sustained attention, relying on both arousal and attention over a period of time, has consistently been associated with activation in a frontal-parietal network, predominantly in the right hemisphere (Pardo et al., 1991; Coull, 1998; Carlsson et al., 2000), a network that was activated to a higher degree in the $U$ conditions.

One other effect of the predictability offered by the visual cue was that the green visual stimulus, which reliably predicted the electric shocks, activated the visual cortex to a higher degree than the blue visual stimulus, in spite of the similar amount of visual input. While the blue square did not carry any such meaning, the green square predicted a motivationally relevant stimulus, and we interpret the increased visual processing in the $\mathrm{C}$ condition to reflect that association with relative significance.

The general idea of this study was to address the question if there is a difference in processing of incoming stimuli under a state of prior knowledge of predictability compared to that of unpredictability. As we wanted to mirror state effects rather than the effects of single events, we used a block design. One limitation of the data in this study is that four out of thirteen subjects in the main experiment rated inconsistently between series and was excluded from further analysis. In the pilot experiment in which the interstimulus interval was shorter and predictability was varied by means of comparing random and non-random (i.e., with constant interstimulus intervals) presentation of the stimuli, all subjects rated the predictable conditions as less painful, less intense and less anxiety provoking. Because these differences in subjective experiences were not well reflected in the fMRI data, probably due to insufficient sensitivity to rapid trains of stimuli (saturation effects), we decided to make the stimulus presentation interval longer. However, longer presentation intervals make it more difficult exactly to predict the coming event by means of the rhythm. Therefore, in the main experiment, we varied the predictability through the introduction of a visual cue that either preceded each of the sensory stimuli with $0.5 \mathrm{~s}$ or was shown at random time points during the stimulation period. Even though the behavioral results in these two experiments were similar, the main experiment did not yield as clear results as the pilot experiment. Several factors seem to have effect on preferences regarding predictability, such as context, anticipatory intervals, the nature of the aversive events, as well as individual differences (Miller, 1981). These factors will affect the strategies to handle the aversive events; two of the strategies referred to as blunting and monitoring (Miller, 1981). Blunting entails cognitive avoidance or transformation of threat relevant information, in which case predictive cues are felt as disturbing and as enhancing anxiety. The monitoring strategy, on the other hand, involves attending to predictive cues, and this may reduce anxiety by defining safe periods or facilitating habituation to the aversive stimulus. Miller (1981) argued that the monitoring mode will be preferred under conditions where the aversive events are invasive and of high frequency, and that blunting will be preferred in situation with the opposite characteristics (i.e., low-frequent non-invasive conditions). In addition, individual variability might also be taken into consideration such that when having a choice, a minority of subjects will choose unpredictability even under conditions apparently not favoring distraction. Similarly, a small minority of subjects chooses predictability under conditions supporting distraction. In this study, the subjects were placed in the camera with little else to attend to than to monitor the presentation of visual and somatosensory stimuli. Stimuli were presented at a relatively high rate and the anticipatory periods were short. We hypothesized that this experimental setup would promote a monitoring strategy. The context in the pilot study with a stimulus presentation rate of $2 \mathrm{~s}$ seemed to support the monitoring mode effectively with lower stress ratings for the predictable rhythmic conditions in all subjects. The lower presentation rate of $4 \mathrm{~s}$ in the main study may have been somewhat less supportive of the monitoring mode allowing for inconsistencies in ratings in some of the subjects.

\section{Conclusions}

We manipulated the stimulus presentation context in terms of predictability of aversive somatosensory events while keeping the input constant. The unpredictable situation induced more anxiety and correlated increases in the anterior insula, a region which has been suggested to provide an interface between interoceptive states and the representation of these states as feelings. This condition also increased the activity in the right fronto-parietal network, which we attribute to enhanced alertness and sustained attention when the aversiveness was unpredictable. Furthermore, we ascribe the involvement of ventral striatum to an active search for any possible temporal structure in the delivery of the electric shocks as 
well as continuous violations in those expectancies. While the unpredictable situation induced more anxiety and increased alertness towards aversiveness, the visual cues in the predictable conditions allowed for correct estimation of the stimulus arrival. Selective attention is known to enhance activity in task-relevant information processing regions. Accordingly, the predictable sensory events were processed to a higher degree in the somatosensory areas of the SI, SII and the posterior insulae. We suggest that the distinct patterns of brain activity in the respective contexts represent two aspects of pain processing; the affective component related to the anterior insula and the $\mathrm{OFC}$ in the unpredictable, more aversive condition, and the sensory-discriminative aspect represented by activity in the SI, SII, and the posterior insulae, during predictability.

\section{Acknowledgments}

This study was supported by grants from The Swedish Research Council (2003-5810), The family Hedlund Foundation and Karolinska Institutet. The project was finished in the context of Stockholm Brain Institute.

\section{References}

Andersson, J.L., Hutton, C., Ashburner, J., Turner, R., Friston, K., 2001. Modeling geometric deformations in EPI time series. NeuroImage 13 (5), 903-919.

Arntz, A., Dreessen, L., De Jong, P., 1994. The influence of anxiety on pain: attentional and attributional mediators. Pain 56 (3), 307-314.

Ashburner, J., Friston, K.J., 1999. Nonlinear spatial normalization using basis functions. Hum. Brain Mapp. 7 (4), 254-266.

Aziz, Q., Schnitzler, A., Enck, P., 2000. Functional neuroimaging of visceral sensation. J. Clin. Neurophysiol. 17 (6), 604-612.

Berlyne, D.E., 1960. Conflict, Arousal and Curiosity. McGraw-Hill, New York.

Berns, G.S., Cohen, J.D., Mintun, M.A., 1997. Brain regions responsive to novelty in the absence of awareness. Science 276 (5316), $1272-1275$.

Bingel, U., Quante, M., Knab, R., Bromm, B., Weiller, C., Buchel, C., 2003. Single trial fMRI reveals significant contralateral bias in responses to laser pain within thalamus and somatosensory cortices. NeuroImage 18 (3), 740-748.

Bolles, R.C., Fanselow, M.S., 1980. A perceptual-defensive-recuperative model of fear and pain. Behav. Brain Sci. 3, 291-323.

Buchel, C., Dolan, R.J., 2000. Classical fear conditioning in functional neuroimaging. Curr. Opin. Neurobiol. 10 (2), 219-223.

Buchel, C., Bornhovd, K., Quante, M., Glauche, V., Bromm, B., Weiller, C., 2002. Dissociable neural responses related to pain intensity, stimulus intensity, and stimulus awareness within the anterior cingulate cortex: a parametric single-trial laser functional magnetic resonance imaging study. J. Neurosci. 22 (3), 970-976.

Carlsson, K., Petrovic, P., Skare, S., Petersson, K.M., Ingvar, M., 2000. Tickling expectations: neural processing in anticipation of a sensory stimulus. J. Cogn. Neurosci. 12 (4), 691-703.

Carlsson, K., Petersson, K.M., Lundqvist, D., Karlsson, A., Ingvar, M., Öhman, A., 2004. Fear and the Amygdala: Manipulation of Awareness Generates Differential Cerebral Responses to Phobic and Fear-Relevant (but Nonfeared) Stimuli. Emotion 4 (4), 340-353.

Coull, J.T., 1998. Neural correlates of attention and arousal: insights from electrophysiology, functional neuroimaging and psychopharmacology. Prog. Neurobiol. 55 (4), 343-361.

Coull, J.T., Vidal, F., Nazarian, B., Macar, F., 2004. Functional anatomy of the attentional modulation of time estimation. Science 303 (5663), $1506-1508$.

Craig, A.D., 2002. How do you feel? Interoception: the sense of the physiological condition of the body. Nat. Rev., Neurosci. 3 (8), $655-666$

Craig, A.D., 2003. A new view of pain as a homeostatic emotion. Trends Neurosci. 26 (6), 303-307.

Critchley, H.D., Wiens, S., Rotshtein, P., Öhman, A., Dolan, R.J., 2004. Neural systems supporting interoceptive awareness (see comment). Nat. Neurosci. 7 (2), 189-195.

Damasio, A.R., Grabowski, T.J., Bechara, A., Damasio, H., Ponto, L.L., Parvizi, J., Hichwa, R.D., 2000. Subcortical and cortical brain activity during the feeling of self-generated emotions. Nat. Neurosci. 3 (10), $1049-1056$.

Davis, M., 1994. The role of the amygdala in emotional learning. Int. Rev. Neurobiol. 36, 225-266.

Devor, M., Seltzer, Z., 1999. Patophysiology of damaged nerves in relation to chronic pain. In: Wall, P.D., Melzack, R. (Eds.), Textbook of pain. Churchhill Livingstone, London.

Domjan, M., 2005. Pavlovian conditioning: a functional perspective. Annu. Rev. Psychol. 56, 179-206.

Elliott, R., Dolan, R.J., Frith, C.D., 2000. Dissociable functions in the medial and lateral orbitofrontal cortex: evidence from human neuroimaging studies. Cereb. Cortex 10 (3), 308-317.

Fanselow, M.S., Helmstetter, F.J., 1988. Conditional analgesia, defensive freezing, and benzodiazepines. Behav. Neurosci. 102 (2), $233-243$

Forkstam, C., Petersson, K.M., 2005. Towards an explicit account of implicit learning. Curr. Opin. Neurol. 18 (4), 435-441.

Friston, K.J., Holmes, A.P., Worsley, K.J., Poline, J.-P., Frackowiak, R.S.J., 1995. Statistical parametric maps in functional imaging: A general linear approach. Hum. Brain Mapp. 2, 189-210.

Friston, K.J., Glaser, D.E., Henson, R.N., Kiebel, S., Phillips, C., Ashburner, J., 2002. Classical and Bayesian inference in neuroimaging: applications. NeuroImage 16 (2), 484-512.

Greitz, T., Bohm, C., Holte, S., Eriksson, L., 1991. A computerized brain atlas: Construction, anatomical content and some applications. J. Comput. Assist. Tomogr. 15, 26-38.

Harville, D.A., 1977. Maximum likelihood approaches to variance component estimation and to related problems. J. Am. Stat. Assoc. 72, $320-338$

Holmes, A.P., Friston, K.J., 1998. Generalisability, random effects and population inference. NeuroImage 7, S754.

Ingvar, M., 1999. Pain and functional imaging (Review, 140 refs). Philos. Trans. R. Soc. London, Ser. B Biol. Sci. 354 (1387), $1347-1358$.

James, W., 1894. Physical basis of emotion. Psychol. Rev. 1, 516-529 (reprinted in Psychological Review 1994;1101:1205-1210).

LaBar, K.S., LeDoux, J.E., Spencer, D.D., Phelps, E.A., 1995. Impaired fear conditioning following unilateral temporal lobectomy in humans. J. Neurosci. 15 (10), 6846-6855.

LaBar, K.S., Gatenby, J.C., Gore, J.C., LeDoux, J.E., Phelps, E.A., 1998. Human amygdala activation during conditioned fear acquisition and extinction: a mixed-trial fMRI study. Neuron 20 (5), 937-945.

Lange, C., 1922. The Emotions. Williams and Williams, Baltimore.

LeDoux, J.E., 2000. Emotion circuits in the brain. Annu. Rev. Neurosci. 23, $155-184$.

Maier, S.F., Watkins, L.R., 1998. Stressor controllability, anxiety, and serotonin. Cogn. Ther. Res. 22 (6), 595-613.

Melzack, R., Casey, K.L., 1968. Sensory, motivational and central control determinants of pain. In: Kenshalo, D.R. (Ed.), The Skin Senses. CC Thomas, Springfield, IL, pp. 423-439.

Mesulam, M.M., Mufson, E.J., 1985. The insula of reil in man and monkey. In: Peters, A., Jones, E.G. (Eds.), Cerebral Cortex Volume 4; Association and Auditory Cortex. Plenum Press, New York, pp. 179-226.

Miller, S.M., 1980. Why having control reduces stress: If I can stop the 
roller coaster, I don't want to get off. In: Garber, J., Seligman, M.E.P. (Eds.), Human helplessness: Theory and applications. Academic press, Inc., New York, pp. 71-96.

Miller, S.M., 1981. Predictability and human stress: Toward a clarification of evidence and theory. In: Berkowitz, L. (Ed.), Advances In Experimental Social Psychology, Vol. 14. Academic press, Inc., New York, pp. 203-256.

Mogg, K., Bradley, B.P., 1998. A cognitive-motivational analysis of anxiety. Behav. Res. Ther. 36 (9), 809-848.

Morris, J.S., Buchel, C., Dolan, R.J., 2001. Parallel neural responses in amygdala subregions and sensory cortex during implicit fear conditioning. Neuroimage 13, 1044-1052.

Nobre, A.C., Coull, J.T., Frith, C.D., Mesulam, M.M., 1999. Orbitofrontal cortex is activated during breaches of expectation in tasks of visual attention. Nat. Neurosci. 2 (1), 11-12.

Öhman, A., 2000a. Anxiety. In: Fink, G. (Ed.), Encyclopedia of Stress, Vol. 1. Academic Press, San Diego.

Öhman, A., 2000b. Fear. In: Fink, G. (Ed.), Encyclopedia of Stress, Vol. 2. Academic Press, San Diego.

Pagnoni, G., Zink, C.F., Montague, P.R., Berns, G.S., 2002. Activity in human ventral striatum locked to errors of reward prediction. Nat. Neurosci. 5 (2), 97-98.

Pardo, J.V., Fox, P.T., Raichle, M.E., 1991. Localization of a human system for sustained attention by positron emission tomography. Nature 349 (6304), 61-64.

Petrovic, P., Kalso, E., Petersson, K.M., Ingvar, M., 2002. Placebo and opioid analgesia- imaging a shared neuronal network. Science 295 (5560), 1737-1740.

Phillips, M.L., Drevets, W.C., Rauch, S.L., Lane, R., 2003a. Neurobiology of emotion perception I: The neural basis of normal emotion perception. Biol. Psychiatry 54 (5), 504-514.

Phillips, M.L., Gregory, L.J., Cullen, S., Coen, S., Ng, V., Andrew, C., Giampietro, V., Bullmore, E., Zelaya, F., Amaro, E., Thompson, D.G., Hobson, A.R., Williams, S.C., Brammer, M., Aziz, Q. 2003b. The effect of negative emotional context on neural and behavioural responses to oesophageal stimulation. (erratum appears in Brain. 2003 May;126 (Pt 5):1248 Note: Cohen S (corrected to Coen S)). Brain 126 (Pt 3):669-684.

Ploghaus, A., Narain, C., Beckmann, C.F., Clare, S., Bantick, S., Wise, R., Matthews, P.M., Rawlins, J.N.P., Tracey, I., 2001. Exacerbation of pain by anxiety is associated with activity in a hippocampal network. J. Neurosci. 21 (24), 9896-9903.

Posner, M.I., Snyder, C.R., Davidson, B.J., 1980. Attention and the detection of signals. J. Exp. Psychol. 109 (2), 160-174.

Rainville, P., 2002. Brain mechanisms of pain affect and pain modulation. Curr. Opin. Neurobiol. 12 (2), 195-204.
Rainville, P., Duncan, G.H., Price, D.D., Carrier, B., Bushnell, M.C., 1997. Pain affect encoded in human anterior cingulate but not somatosensory cortex. Science 277 (5328), 968-971.

Rees, G., Frith, C.D., 1998. How do we select perceptions and actions? Human brain imaging studies. Philos. Trans. R. Soc. London, Ser. B, Biol. Sci. 353 (1373), 1283-1293.

Rees, G., Frackowiak, R., Frith, C., 1997. Two modulatory effects of attention that mediate object categorization in human cortex. Science 275 (5301), 835-838.

Rescorla, R.A., 1967. Pavlovian conditioning and its proper control procedures. Psychol. Rev. 74, 71-80.

Rhudy, J.L., Meagher, M.W., 2000. Fear and anxiety: Divergent effects on human pain thresholds. Pain 84 (1), 65-75.

Rolls, E.T., 2004. The functions of the orbitofrontal cortex. Brain Cogn. 55 (1), 11-29.

Schultz, W., 2002. Getting formal with dopamine and reward. Neuron 36 (2), 241-263.

Schultz, W., Tremblay, L., Hollerman, J.R., 2000. Reward processing in primate orbitofrontal cortex and basal ganglia. Cereb. Cortex 10 (3), $272-284$.

Selemon, L.D., Goldman-Rakic, P.S., 1985. Longitudinal topography and interdigitation of corticostriatal projections in the rhesus monkey. J. Neurosci. 5 (3), 776-794

Seligman, M.E.P., 1968. Chronic fear produced by unpredictable electric shock. J. Comp. Physiol. Psychol. 66, 402-411.

Seymour, B., O’Doherty, J.P., Dayan, P., Koltzenburg, M., Jones, A.K., Dolan, R.J., Friston, K.J., Frackowiak, R.S., 2004. Temporal difference models describe higher-order learning in humans. Nature 429 (6992), $664-667$.

Seymour, B., O’Doherty, J.P., Koltzenburg, M., Wiech, K., Frackowiak, R., Friston, K., Dolan, R., 2005. Opponent appetitive-aversive neural processes underlie predictive learning of pain relief. Nat. Neurosci. 8 (9), 1234-1240.

Shulman, G.L., Corbetta, M., Buckner, R.L., Raichle, M.E., Fiez, J.A., Miezin, F.M., Petersen, S.E., 1997. Top-down modulation of early sensory cortex. Cereb. Cortex 7 (3), 193-206.

Singer, T., Seymour, B., O’Doherty, J., Kaube, H., Dolan, R.J., Frith, C., 2004. Empathy for pain involves the affective but not sensory components of pain. Science 303, 1157-1162.

Talairach, J., Tournoux, P., 1988. Co-Planar Stereotaxic Atlas of the Human Brain. George Thieme Verlag, Stuttgart.

Vogt, B.A., Pandya, D.N., 1987. Cingulate cortex of the rhesus monkey: II. Cortical afferents. J. Comp. Neurol. 262 (2), 271-289.

Walker, D.L., Toufexis, D.J., Davis, M., 2003. The role of the bed nucleus of the stria terminalis versus the amygdala in fear, stress and anxiety. Eur. J. Pharmacol. 463 (1-3), 199-216. 